CIUDAD Y TERRITORIO

ESTUDIOS TERRITORIALES

ISSN(P): 1133-4762; ISSN(E): 2659-3254

Vol. LII, № 203, primavera 2020

Págs. 57-70

https://doi.org/10.37230/CyTET.2020.203.05

CC BY-NC 4.0

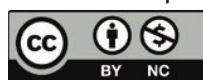

\title{
Aplicación de la perspectiva de género al urbanismo y la arquitectura. Experiencias a escala regional y municipal en Cataluña
}

\author{
Zaida Muxí-Martínez
}

Doctora arquitecta

Departamento de Urbanismo y Ordenación del Territorio (DOUT) Escuela Técnica Superior de Arquitectura de Barcelona (ETSAB) Universidad Politécnica de Cataluña-BarcelonaTech (UPC)

RESUMEN: La aprobación de la Ley 2/2004, de 4 de junio, de mejora de barrios, áreas urbanas y villas que requieren una atención especial, ha marcado un antes y después en la visibilización y aplicación de la perspectiva de género en urbanismo en Cataluña. En los años siguientes ha continuado la apuesta por introducir el género en otras leyes de alcance regional, con un desarrollo transversal en la política catalana. Este artículo se centra en la experiencia de las políticas urbanísticas en Barcelona y se reseñan algunos ejemplos de otras ciudades: Santa Coloma de Gramenet, Girona, Garraf y Olesa. No solo desde las normativas o la incorporación de la participación ciudadana con perspectiva de género, sino también desde otros ámbitos como la cultura o los eventos de representación política, se ha buscado la paridad de representación, dando un mayor protagonismo a las mujeres para compensar los años de invisibilidad de sus aportaciones. Estos cambios, que están enraizados en los movimientos y luchas feministas de décadas, no son acciones solitarias de gobierno, sino reflejo de una sociedad que aspira a la igualdad real.

PALABRAS CLAVE: Cataluña. Perspectiva de género. Urbanismo. Feminismo.

\section{Applying gender perspective to urban planning and architecture. Experiences at regional and municipal level in Catalonia}

ABSTRACT: The approval of Law 2/2004, of June 4, on the improvement of neighborhoods, urban areas and towns requiring special attention, has marked a turning point in the visibility and application of the gender perspective in urban planning in Catalonia. In the following years, the commitment to introduce gender into other laws of regional scope has continued, with a transversal development in Catalan politics. This article focuses on the experience of urban policies in Barcelona and some examples from other cities are described:

Recibido: 04/09/.2019; Revisado: 18.11.2019

Correo electrónico: zaida.muxi@upc.edu～Nº ORCID: https://orcid.org/0000-0002-8438-3824 
Santa Coloma de Gramenet, Girona, Garraf and Olesa. Not only from the regulations or the incorporation of citizen participation with a gender perspective, but also from other areas such as culture or political representation events, representation parity has been sought, giving women greater prominence to compensate years of invisibility in the past. These changes, which are rooted in the feminist movements and struggles of decades, are not solitary actions of government, but a reflection of a society aspiring for real equality.

KEYWORDS: Catalonia. Gender perspective. Town planning. Feminism.

\section{Introducción}

a aprobación de la ley de barrios en el año 2004 (Ley 2/2004, de 4 de junio, de mejora de barrios, áreas urbanas y villas que requieren una atención especial) ha marcado un antes y después en la visibilización y aplicación de la perspectiva de género en urbanismo en Cataluña. El punto 6 de la Ley estableció que las rehabilitaciones de barrios garantizarían "la equidad de género en el uso del espacio urbano y de los equipamientos".

En los años siguientes ha continuado la apuesta por introducir el género en otras leyes de alcance regional ligadas al medioambiente construido, debido a la labor conjunta entre el Instituto Catalán de las Mujeres (ICD) y el Departamento de Política Territorial y Obras Públicas de la Generalitat de Cataluña entre 2004 y 2010, del Plan de acción y desarrollo de las políticas de las mujeres en Cataluña (2005-2007) y el Plan de políticas de mujeres del Gobierno de la Generalitat de Cataluña (2007-2011), las acciones tuvieron un desarrollo transversal en la política catalana.

La Llei 18/2007, de 28 de desembre, del dret a l'habitatge, en su artículo 23 sobre los requisitos exigibles a las viviendas, establece que en los procesos de edificación, conservación y rehabilitación se han de fijar criterios de género, por medio de nuevos tipos de vivienda que tengan en cuenta los diferentes modelos de convivencia, así como las necesidades de grupos específicos; el punto d) de dicho artículo, explica que la innovación en la concepción y el diseño, debe permitir la flexibilidad en el uso de elementos para facilitar el trabajo doméstico y para adecuarse a los nuevos roles de género, consintiendo las transformaciones dentro de la vivienda para adaptarlo a las variaciones de las estructuras familiares.

De una etapa normativa, se pasó a experiencias en las ciudades que obtuvieron la financiación mediante la Ley de Barrios generando una repercusión ampliada por la acción, la formación y la difusión que se generaron.
En el período 2015-2019 los gobiernos de diversas ciudades han impulsado políticas de género $y$ feministas que han impactado de manera transversal en las políticas municipales influyendo en los proyectos urbanos. Este artículo se focaliza en la experiencia de Barcelona y se reseñan algunos ejemplos de otras ciudades.

\section{Recorrido de la perspectiva de género aplicada al urbanismo en Cataluña y Barcelona}

Las luchas feministas por ciudades más justas, inclusivas y que tengan en cuenta las tareas de la reproducción y los cuidados de la vida, llevan décadas en nuestro contexto, aunque ha tenido que pasar bastante tiempo para que se hayan comenzado a ver reflejadas en las legislaciones y acciones concretas.

En la última década de la dictadura de Francisco Franco (1939-1975), en 1964, se aprobó la Ley de las Asociaciones que legalizó las asociaciones de vecinos, significando, no por voluntad del régimen, un primer espacio democrático y de pensamiento urbano. Estas asociaciones contaron con vocalías de mujeres que trabajaron en visibilizar las dificultades de la vida cotidiana en la ciudad tardofranquista, reclamando mejoras urbanas, servicios, equipamientos, y espacios públicos para la vida cotidiana (Muxí, 2009). Este reclamo es consecuencia de la división del trabajo basada en roles de género, ya que debido a ello las mujeres son quienes mayoritariamente se han hecho y hacen cargo de las tareas reproductivas que las provee de un conocimiento amplio de las necesidades urbanas de las que el urbanismo tradicional está muy alejado. En 1970, se realizó en el barrio obrero del Besós un estudio para conocer las necesidades de guarderías, determinando que el $22 \%$ de las mujeres trabajaban fuera del hogar y un $50 \%$ trabajaba a cambio de un salario desde sus propios hogares. Es obvio aclarar que entonces apenas existían servicios colectivos o sociales que permitieran a las mujeres liberarse de las obligaciones de la crianza y los cuidados (Muxí, 2009). 
Los movimientos feministas adquirieron una gran relevancia y visibilidad en los años de la transición defendiendo la incorporación de la vida cotidiana en la práctica urbanística. Los grupos feministas (MAGRO, 2014) organizaron las "Primeras Jornadas por la Liberación de la Mujer" en Madrid en diciembre de 1975 y, en marzo de 1976, se celebraron en Barcelona las "I Jornades Catalanes de la Dona". En las jornadas de Barcelona se reunieron unas tres mil personas con representación de mujeres de toda España y participación del movimiento vecinal, a través de la asistencia de diez asociaciones de vecinos y vecinas, y diecinueve vocalías de mujeres de asociaciones de vecinos y vecinas de Cataluña.

Una de las nueve ponencias presentadas, dentro del apartado Ponencias, Comunicaciones y Conclusiones, fue "Mujer y Barrios" donde se demostraba y visibilizaba la relación existente entre las mujeres y su entorno habitable teniendo en cuenta que éste era el lugar por donde transcurre su vida cotidiana. Junto a la ponencia se presentaron una serie de comunicaciones que trataban el mismo tema y lo completaban desde distintos puntos de vistas y experiencias

En la ponencia "Mujer y barrios", [...] se destacaban dos cuestiones importantes: [...]

"Las malas condiciones de vida en los barrios afectan sobre todo a las mujeres ya que son ellas las que más tiempo pasan y más tareas realizan en ellos". [y] "El problema de participación ciudadana es más grave en la mujer porque se encuentra en inferioridad, debido a la opresión a la que está sometida y a su exclusión del espacio público, con respecto al hombre". (MAGRO, 2014: 115-116)

La revista Vindicación feminista (1976-1979) publicó a partir del número 4, de octubre de 1976, una nueva sección "La mujer en los barrios" que tal como explicaba Empar Pineda tenía el objetivo de dar a conocer de la mejor manera las condiciones de vida de cientos de miles de mujeres en los barrios, en donde se manifestaba claramente la opresión que sufrían las mujeres, y cómo la vida cotidiana resultaba discriminatoria (Muxí, 2009:5).

En 1980 se celebraron las I Jornadas de Feministas Independientes en Barcelona, en las que Anna Bofill Levy propuso la conferencia "Mujer y arquitectura", una mirada diferente de las mujeres sobre el entorno poniendo de manifiesto la relación entre estructura patriarcal y la forma de nuestras ciudades (BoFILL, 2013).

En 1995 Isabel Segura publicó la Guía de Mujeres de Barcelona, en la que descubre las huellas de los pasos de las mujeres por la ciudad desde la época romana hasta la actualidad, espacios prohibidos y espacios vividos, leyendo la historia urbana desde otro lugar, haciendo visible lo que se encuentra escondido por el monumento, aquello que se encuentra en los márgenes de la historia tradicional.

En Europa en 1995 se estableció un nuevo marco político con la Carta Europea de las Mujeres en la Ciudad, coincidiendo en ese mismo año con la Conferencia de Mujeres de Naciones Unidas de Beijin 1995, en la que se establecieron acuerdos para dar prioridad y alentar las políticas de género. La Carta Europea aboga por la incorporación de la perspectiva de género y la participación de las mujeres en 5 áreas: planeamiento urbano y desarrollo, movilidad, seguridad, vivienda y procesos de toma de decisiones políticas. Esta Carta fue ratificada al tiempo que se firmaba la Carta del Derecho de las Mujeres a la Ciudad, en el Fórum Internacional de Mujeres celebrado en Barcelona en el año 2004. En nuestro campo de conocimiento, es decir, la ciudad y el urbanismo, fueron inspiración para esta ratificación los ejemplos de Viena con la Oficina de Planeamiento urbano y género creada en 1998 y dirigida por la urbanista Eva Kail, y el programa de Montreal "Mujeres y ciudades", enfocado en la mejora de la seguridad de las mujeres y la vida cotidiana a través de la planificación urbana, y cuyas observaciones y experiencias fuero recogidos, con la coordinación de Anne Michaud, en Pour un environment urbain sécuritaire. Guide d'aménagement. Programme Femmes et Ville de la Ville de Montreal, del 2002.

Un precedente, temporalmente posterior a los ya citados, que también ha influido en la ciudad de Barcelona, ha sido la Red Mujer y Hábitat de América Latina, especialmente a través del Programa Regional "Ciudades sin violencia hacia las Mujeres, Ciudades seguras para tod@s"que ha sido realizado entre 2006 y 2011 , y que ha trabajado en la implementación de políticas urbanas con perspectiva de género para mejorar la seguridad de las mujeres.

En el contexto catalán, Anna Bofill Levy, Rosa Maria Dumenjo Martí e Isabel Segura Soriano realizaron con fondos europeos y para la Fundació Maria Aurèlia Capmany el proyecto "Mujeres y ciudad" con el objetivo de incorporar sus necesidades y deseos en el planeamiento urbano. En 1998, como resultado del proyecto publicaron el "Libro blanco. Las mujeres y la ciudad" o "Manual de Recomendaciones para la concepción de los aglomerados urbanos desde la perspectiva de 
género" que compiló recomendaciones para la redacción de políticas de transformación de las ciudades catalanas. Este trabajo creó y fortaleció una red de profesionales e instituciones, tanto a escala local como internacional, para abogar por la incorporación de la perspectiva de género en las cuestiones urbanas. Esta red estuvo formada por ciudades (Barcelona, Lleida, Reus, Donosti, Arbeit und Leben de Sachsen Anhal); la provincia de Barcelona; la comarca del Garraf; el Institut Català de la dona ${ }^{1}$; el Consejo de las Mujeres de la Comunidad de Madrid; el VES (Emancipatie Bureau Zuid-Holland); la red "Quartiers en Crise" y la red de ciudades educadoras (Ortiz Escalante, 2019: 120).

En 1999 se realizó en Barcelona el primer Congreso de las Mujeres de Barcelona en el que participaron los consejos de mujeres de los diez distritos y en el que se realizó la ponencia "El espacio urbano, los tiempos y las mujeres" donde se hicieron propuestas sobre vivienda, espacio urbano, accesibilidad y seguridad urbana.

En estas jornadas se presentaron propuestas respecto a los usos del tiempo, la ciudad y las personas, que encontraron eco en el programa "Barcelona Bonanit" iniciado a mediados de los 90, que incidía en la organización social de las personas jóvenes y buscaba la igualdad de oportunidades y la democratización de la cultura. De estos planteamientos surgieron las primeras actuaciones como la remodelación de los horarios de las bibliotecas y del transporte público, así como algunos recorridos, cargas y descargas silenciosas en la noche y el cambio gradual de los horarios de los mercados. El objetivo final de las políticas públicas de nuevos usos sociales del tiempo era conseguir un mayor bienestar y más equidad entre hombres y mujeres. Desde este programa se encargaron una serie de estudios ${ }^{2}$ que permitieran avanzar en esta política de tiempos, liderada por la regidora de Nuevos Usos Sociales del Tiempo (NUST) Imma Moraleda, siendo la primera publicación, en 2006, "Les politiques del temps: un debat obert" realizada por Teresa Torns, Vicent Borras, Sara Moreno, Carolina Recio del QUIT (Centre d'Estudis Sociològics sobre la Vida Quotidiana i el Treball):

\footnotetext{
${ }^{1}$ Fue durante el gobierno de Pasqual Maragall al frente de la Generalitat, que la dirección colegiada del Institut con Marta Selva i Masoliver, presidenta, Mercé Fernández i Gesalí, vicepresidenta, Anna Solà i Arguimbau, Gerenta y la Directora de programas Isabel Segura i Soriano (2003-2006) cambió su nombre, transformando el singular "dona" por el plural "dones".
}

"La creación del NUST tiene otros antecedentes en el gobierno municipal. En el año 1994 se puso en marcha una experiencia piloto sobre el tiempo y la ciudad [...] las experiencias que se pusieron en marcha incidían, principalmente, en el refuerzo de la política de descentralización de los servicios municipales, para que sean más próximos a los ciudadanos y ciudadanas [...] más flexibilidad horaria en las escuelas bressol y primarias. Se exploraron posibilidades de ofrecer flexibilidad laboral a funcionarios y funcionarias municipales para poder compatibilizar los horarios laborales con el tiempo del trabajo de cuidado de las personas [...] Desde el inicio de las primeras actuaciones en Sants hasta ahora, el proyecto que más se ha extendido por la ciudad es el de Bancos del Tiempo [...] experiencia que comenzó a desarrollarse a finales de 1990 [...] fueron pensados para hacer visibles y dar valor a las tareas que cotidianamente hacen las mujeres procurando el bienestar cotidiano.. El objetivo siempre ha sido el intercambio de tiempo, lejos de la lógica mercantil y, subsidiariamente, construir redes de solidaridad entre ciudadanos y ciudadanas [...] redes que promuevan en la ciudad valores comunitarios que no suelen estar presentes" (ToRNs, 2006: 47-48)

En los primeros años del milenio comenzaron a realizarse congresos de mujeres en diferentes comarcas de Cataluña, como el del Baix Llobregat, en donde se celebró su primera edición en marzo de 2002 y la quinta en 2018. Para ello se ha contado con el apoyo de la Diputación de Barcelona. Estos congresos incorporan la discusión urbana desde la experiencia de las mujeres que habitan los territorios, así como de las experiencias desde los feminismos para la mejora de las condiciones de vida en las ciudades.

El proyecto "Mujeres y Ciudad" ha marcado un hito en la historia del planeamiento urbano feminista en Cataluña, e influenció en las políticas y leyes que comenzaron a desarrollarse a inicios de los años 2000, especialmente las que se aprobaron en el primer gobierno tripartito de izquierdas (2003-2006), después de 25 años de gobiernos conservadores en Cataluña, debido a que en este gobierno el Institut Català de les Dones fue colocado orgánicamente como dependiente directo de presidencia, por lo que la perspectiva de género permeó transversal-

Dando cuenta de la diversidad de las mujeres, rechazando la idea de grupo homogéneo.

${ }^{2}$ Los "Dossiers del temps" recogen una interesante producción en torno a los usos del tiempo

http://ajuntament.barcelona.cat/tempsicures/ca/canal/dossiersdel-temps 
mente todas las políticas de este período. En particular la conocida como Ley de Barrios (Llei de millora de barris, àrees urbanes $i$ viles que requereixen una atenció especial, de 26 de mayo de 2004), primera normativa del ámbito urbanístico en el Estado Español que introdujo el género como un parámetro del planeamiento urbano.

La ley de Barrios estaba cofinanciada al 50\% por la Generalitat y por cada municipio. Se establecieron tres tipos de tejidos urbanos prioritarios, cascos históricos, polígonos residenciales y barrios con origen en la autoproducción; el sistema de selección de los proyectos establecía una serie de criterios sociales, económicos y físicos que no eran absolutos, sino que se debían analizar en relación a su propio contexto. La ley exigía que los proyectos abordaran 8 criterios, todos ellos de obligado cumplimiento, siendo el punto 6 , el de equidad de género en el uso de los espacios públicos y equipamientos. Es a raíz de esta ley que muchos gobiernos municipales comenzaron a implementar la perspectiva de género en el planeamiento y el proyecto urbano.

Esta ley generó la necesidad de conocimiento y, por lo tanto, de formación en la materia que fue y está especialmente articulada por la Diputación de Barcelona, a través de cursos sobre urbanismo y género, dirigidos al personal técnico municipal. Algunas ciudades de las otras provincias catalanas realizan desde entonces formaciones específicas para su personal técnico. Así mismo, desde el ICD se ofrecieron, en coincidencia con la aplicación de la Ley de barrios, cursos de formación para las asociaciones de mujeres del territorio catalán. Se trataban de prácticas cortas que tenían como objetivo acercar el urbanismo a las mujeres, desvelando el conocimiento que entraña su experiencia cotidiana en las ciudades y pueblos. En coincidencia con este momento se organizaron en Barcelona, en abril del 2005, las Jornadas Urbanismo y Género. Una visión necesaria para todos, dirigidas por Isabela Velázquez, que generaron un gran impacto académico, político y mediático, y contaron con la participación de académicas, técnicas y políticas tanto nacionales como extranjeras de un amplio abanico profesional, convirtiéndose en un referente histórico.

También permitió introducir el género como una categoría para el proyecto urbano y para la docencia, como ha sucedido en la Escuela Técnica Superior de Arquitectura de Barcelona donde en el curso 2010-2011 yo misma impartí en el doctorado del departamento de urbanismo, y por primera vez, la materia de Urbanismo y género. En octubre 2011 se celebraron en la ETSAB las jornadas "Estudios urbanos, género y feminismo: Teorías y experiencias" organizadas por Blanca G. Valdivia y Adriana Ciocoletto, del Col•lectiu Punt 6, como doctorandas de la UPC contando con el apoyo de la Oficina de Soporte a la Igualdad de oportunidades de la UPC y una subvención del Institut Catalá de les Dones. Progresivamente se ha incorporado en la docen-cia esta perspectiva resaltando especialmente las tesis doctorales de Tania Magro, Adriana Ciocoletto, Daniela Arias y Blanca Valdivia.

La ley de barrios fue precursora en la incorporación de la perspectiva de género en el planeamiento urbano siguiéndole en el 2005 el Texto refundido de la Ley de Urbanismo, en el decreto Legislativo 1/2005 de la Ley de los Planes de Ordenación Urbanística Municipal, por la que se requiere que estos planes incorporen evaluaciones del impacto de género de las propuestas urbanas. La ley modificada en 2012 estipula que el Departamento de Territorio y Sostenibilidad tiene que incluir la perspectiva de género para garantizar la igual representación de hombres y mujeres en los órganos colegiados de planeamiento. Así la Ley Catalana de Igualdad de 2015 incorpora la perspectiva de género en urbanismo (Llei 17/2015, del 21 de juliol, d'igualtat efectiva de dones i homes) y establece que todas las políticas de planeamiento urbano, vivienda, movilidad y sostenibilidad incluyan la perspectiva de género en todas sus fases, del diagnóstico a la evaluación. Para poder cumplir con estas medidas, la administración pública tiene que garantizar: el acceso a la formación en cuestiones de género del personal técnico y político; segregación de datos estadísticos por sexo; la elaboración de estudios de impactos de género y la definición de medidas correctoras; la aplicación de políticas de planeamiento urbano para crear ciudades compactas con mezcla de usos y que respondan en proximidad a las necesidades cotidianas de las personas; creación de programas que faciliten el acceso a las mujeres a la vivienda y políticas de movilidad que den prioridad a las actividades de la vida cotidiana, especialmente aquellas relacionadas con los trabajos domésticos y de cuidados.

Todos los esfuerzos, luchas y leyes van dando poco a poco sus frutos, y actualmente (2019) se requieren personas técnicas expertas en género para concursos de grandes proyectos urbanos, así como para la redacción y revisión 
de POUMs. Tal es el caso del trabajo realizado por el Col•lectiu Punt 6, que trabaja en urbanismo feminista aplicando la perspectiva de género interseccional desde 2005 , que ha visto como la demanda de colaboraciones, así como el interés en su trabajo y en los cursos de formación han crecido exponencialmente los últimos años, especialmente en el período 2015-2019. Y esto es, en parte, debido al apoyo explícito a políticas feministas realizado por alcaldesas de varios municipios de la región, y que se ha visto especialmente reforzado por las dos principales ciudades españolas. En Madrid, Manuela Carmena ha abogado por una ciudad de las personas y en Barcelona, Ada Colau lo ha hecho por una ciudad feminista y cuidadora.

\section{Políticas y urbanismo feministas en Barcelona}

No siempre la aplicación práctica de las leyes ha sido rápidamente implementada, ya que hay resistencias tanto a nivel técnico como académico. Sin embargo, los movimientos de base feministas han estado empujando, como hemos visto, estas políticas que, tras un fuerte descenso en su aplicación en el período 2011-2015, se ha visto reimpulsado por el gobierno de Barcelona en Comú en el período 2015-2019.

Este gobierno de Barcelona, encabezado por Ada Colau, ha tenido por motor y base de su política los feminismos. Esto se ha visto reflejado desde la candidatura que fue conformada por una lista en cremallera (alternancia de mujer-hombre) y que, evidentemente, ha estado por primera vez encabezada por una mujer. Áreas de relevancia política han estado dirigidas por mujeres, como la tenienta de alcaldesa Janet Sanz, quien ha estado a cargo de Urbanismo, Ecología urbana y Movilidad.

La presencia de mujeres en puestos de poder no es suficiente para implementar políticas feministas. Por lo tanto, en este contexto ¿Qué ha significado la feminización feminista ${ }^{3}$ de la política?

La primera cuestión simbólica e importante es que por primera vez la alcaldía estuviera ocupada

\footnotetext{
${ }^{3}$ Esta expresión de aparente duplicación de términos no lo es tal, ya que por un lado está la presencia igualitaria de representación según sexos, y que, debido a la novedad de la incorporación de las mujeres, se denomine feminización de la política
}

por una mujer y, en este caso, la molestia de su presencia en estamentos de poder se ha visto acrecentada por encarnar una doble alteridad, de sexo y de clase. La propia alcaldesa ha denunciado las formas y tiempos patriarcales de la política, un cambio que evidentemente aún está pendiente, ya que no se trata solo de una reorganización de los gobiernos, sino de la sociedad en su conjunto, en la que no predomine la esfera productiva por encima de la reproductiva, la sociopolítica y la personal. Las formas patriarcales de organización del tiempo y el espacio son las que han hecho que ciertas personas solo se tengan que dedicar al trabajo productivo, no permitiendo ni considerando los tiempos de los cuidados, los personales ni los socio-políticos-comunitarios; y esto ha sido así porque han existido otras personas, mayoritariamente mujeres, que dedican y han dedicado su tiempo, muchas veces de manera gratuita, a las tareas de la reproducción. Por lo que una primera dificultad para la aplicación de unas políticas feministas, que pongan en el centro los cuidados, es para quienes las impulsan, ya que la organización política no permite poner en el centro sus propios cuidados. A un ser humano político se le exige la deshumanización.

Como declaración de intenciones y de orientación del gobierno fue creada la regiduría de Feminismos y Diversidad Sexual y de Género, a cargo de Laura Pérez Castaño, desde donde se imprimió un carácter transversal de los feminismos y la perspectiva de género a todas las políticas. Muestra de ello es la implementación del Plan de la Justicia de Género 2016-20204, en el que se requiere la incorporación de la perspectiva feminista en todas las políticas municipales, incluyendo planeamiento, urbanismo y seguridad. Este Plan estructurado en cuatro ejes: cambio institucional; economía de la vida y organización del tiempo; la ciudad de los derechos y barrios habitables e inclusivos, es el marco para el desarrollo de las posteriores normativas, políticas y proyectos que se han aprobado y desarrollado en el mandato, como la medida de gobierno "Urbanismo con perspectiva de género. El urbanismo de la vida cotidiana" que se explica más adelante.

Desde diferentes ámbitos del gobierno se ha trabajado con políticas feministas transversales,

y por otro está la práctica política desde los feminismos que es lo que ha encarnado el primer gobierno de Barcelona en Comú ${ }^{4}$ https://ajuntament.barcelona.cat/feminismes-lgtbi/sites/default/ files/documentacio/p 4.2 pla per la justicia de genere $0 . p d f$ 
como las llevadas a cabo por la segunda tenienta de alcaldesa Laia Ortíz Castelví desde el área de derechos sociales, recuperando guarderías municipales privatizadas y creando nuevas, así como estableciendo un sistema de pago progresivo en función de la renta familiar. Estos equipamientos son básicos para la igualdad de oportunidades, dado que las diferencias sociales y de oportunidades se establecen desde la primera infancia y para que las mujeres-madres tengan igualdad en acceso a derechos es imprescindible el acompañamiento en los cuidados a la infancia. A finales de 2018 Barcelona inauguró su guardería (escola bressol) número 100 (BLANCHARD, 2018), contando así con una de las redes más grande de Europa en la que se atiende a 8400 infantes, y aun así es una red insuficiente. Esta red de escuelas "bressol" municipales tiene dos procedencias: una la absorción por parte del ayuntamiento en 1991 de los centros privados o de los creados por cooperativas familiares durante la Transición, y dos, las construidas por el propio ayuntamiento desde el año 2000. Sin embargo, el reconocimiento oficial de la etapa educativa desde el nacimiento, de ahí la palabra bressol, cuna en castellano, que se utiliza para denominarla, comenzó durante el período republicano, en los años 30 del siglo XX (BASTARDES, 2018).

La concejalía de vivienda, a cargo de Josep Maria Montaner, fue reorganizada interna y externamente. Fue colocada en la estructura políticaorganizativa en derechos sociales, marcando un cambio de concepto importante: la vivienda no es más entendida como un elemento meramente edificado y de plusvalía urbana, sino como un derecho. El Plan por el derecho a la vivienda en Barcelona 2016-2025 fue redactado y aprobado al comienzo del mandato con el objetivo de ampliar el parque de vivienda social asequible y diversificando los sistemas de obtención y tenencia. No solo hacer nuevas viviendas, sino la compra de edificios para sacarlos del mercado especulativo que está expulsando a la ciudadanía fue lo que llevó a ampliar la tarea de la promoción de vivienda a la rehabilitación de las mismas. El antiguo Patronato de la Vivienda ha sido transformado en el Instituto Municipal de la Vivienda y la Rehabilitación de Barcelona (IMHAB). La ampliación de los tipos de tenencia ha incorporado la vivienda cooperativa en sesión de uso. Y la mayor parte de vivienda lograda, ya sea por la vía de la obra nueva, de compra, de cesión de bancos, etc. pasa a ser mayoritariamente de alquiler asequible, siendo uno de los objetivos del gobierno no perder patrimonio público. La vivienda también es entendida y atendida desde el planeamiento, y por ello, y con el objetivo de no concentrar la vivienda asequible en determinados barrios, se ha aprobado que el $30 \%$ de nuevas promociones de vivienda nueva o rehabilitación integral, que superen los $600 \mathrm{~m}^{2}$, sea para alquiler asequible.

El Plan por el derecho a la vivienda 2016-2025 ha incorporado específicamente la perspectiva de género en las actuaciones relacionadas con la formación del personal de las oficinas de vivienda y los criterios de género en el diseño de nuevos modelos de vivienda de protección oficial y en las dotacionales, para incluir tipologías más flexibles y diversas que garanticen la adaptación a lo largo del ciclo de vida y a los diferentes perfiles de las personas solicitantes. También propone explícitamente que las viviendas no sean androcéntricas y que incluyan espacios de vida comunitaria, juegos infantiles, espacios luminosos y seguros, entre otros. Se incorpora la perspectiva de género en los baremos para adjudicar viviendas, teniendo en cuenta la vulnerabilidad especial de las mujeres, especialmente entre la gente mayor y la juventud (FALAGAN, 2019).

Es importante señalar que al tiempo que se redacta el Plan de la vivienda se está redactando la Llei 4/2016, del 23 de desembre, de mesures de protecció del dret al'habitatge de les persones en risc d'exclusió residencial, que fija a nivel catalán algunas consideraciones en las que también ha trabajado el Plan por el derecho a la vivienda 2016-2020 de Barcelona. Específicamente en el Artículo 8, punto f se establece:

"Aplicar la perspectiva de género e incorporar la perspectiva de las mujeres en todos los niveles y en todas las etapas de las actuaciones que se lleven a término para proteger el derecho a la vivienda de las personas que se encuentran en situación de exclusión residencial o que estén en riesgo de encontrarse".

Desde la regiduría de vivienda se ha promovido una nueva tipología de vivienda pública colectiva denominado Co-Habitatge (co-vivienda o cohousing) o vivienda cooperativa en cesión de uso. Hacía unos años que grupos cooperativos organizados por el derecho a la vivienda estaban trabajando en dos proyectos: el de la cooperativa La Borda en Can Batlló, que se inició con la idea de rehabilitar una nave como vivienda colectiva con usos y espacios compartidos, y que había llegado al finalizar el mandato 2011-2015 a un acuerdo verbal para un solar para obra nueva en 
el recinto de Can Batlló, y el de la calle Princesa, que era para rehabilitar un edificio de propiedad municipal para 6 viviendas de la cooperativa Sostre Cívic. En el mandato 2015-2019 se establecieron las bases reglamentarias y legales para hacer posible este sistema de viviendas, firmando como primer paso la cesión de uso del solar en la avenida Constitución para La Borda, habitado desde diciembre 2018 y el edificio en la calle Princesa, habitado desde finales de 2017.

El sistema de viviendas cooperativas de tenencia colectiva en cesión de uso con espacios compartidos es una solución que coloca los cuidados en el centro, ya que los espacios compartidos buscan socializar (LACOL \& La ciutat invisible, 2018) acompañar y hacer más ligera la carga derivada de los cuidados del hogar y de las personas dependientes.

"[...] en muchas de las cooperativas se socializan estas prácticas invisibilizadas e históricamente feminizadas, como el cuidado de menores y de personas dependientes, la alimentación, la salud o la higiene.

En esta línea, cada vez más proyectos cooperativos incorporan la perspectiva de género en su desarrollo. [...] También La Borda se autodefine como un proyecto feminista, poniendo énfasis en las tareas de cuidados y diseñando sus viviendas según criterios de distribución no jerárquica de los espacios". (LACOL, 2018:104).

En el año 2018 se lanzó el concurso BCN-NYC Affordable Housing Challenge ${ }^{5}$ para buscar tecnologías y herramientas innovadoras que disminuyan los tiempos y costos de construcción y la rehabilitación de zonas urbanas densas con la finalidad de hacer la vivienda más asequible para todas las personas. Entre las tres propuestas finalistas estuvo la presentada por el Col•lectiu punt 6 que propuso desde una perspectiva feminista compartir zonas comunes formando una red territorial de viviendas accesibles y asequibles con el objetivo de colectivizar el trabajo doméstico, asistencial y comunitario, así como espacios para el trabajo productivo. Siendo su potencial la reducción de costos de vida, manteniendo la accesibilidad a la vivienda asequible, protegiendo el derecho a la vivienda y a la ciudad.

\footnotetext{
${ }^{5}$ Organizado por el programa i.lab del comisionado de Tecnología e innovación Digital, la Regiduria de Vivienda y rehabilitación del Ayun-
} tamiento de Barcelona y el gobierno de la ciudad de Nueva York.
Por su parte, el Instituto Metropolitano de Promoción de Suelo y Gestión Patrimonial (IMPSOL) siguiendo las recomendaciones del artículo 23 de la ley $18 / 2007$, ha recogido criterios de género en los pliegos técnicos para concursos de vivienda. Estos pretenden viviendas inclusivas y diseñadas teniendo en cuenta la perspectiva de género, rompiendo con las jerarquías espaciales, con los repartos de roles y de poder tradicional. Las viviendas considerarán la incorporación de la diversidad de necesidades en diferentes etapas de la vida, con especial atención a las tareas de cuidados. Se establece la necesaria consideración de espacios de guardados, teniendo en cuenta la cadena de la ropa, de la alimentación, la basura y el reciclaje. Estos criterios también tienen que estar reflejados en los espacios comunitarios para que sean seguros e inclusivos, evitando rincones sin visibilidad.

También el IMPSOL establece como tipología genérica un espacio principal de comedor-estar y que la cocina puede ser segregada o no. En caso que no lo sea se procurará que el diseño permita cerrarla para poder no ser vista cuando se disfruta del descanso en la sala. Las 2 o 3 habitaciones no tienen que tener jerarquía de superficie.

El 22 de marzo de $2017^{6}$ se aprobó, impulsada por la Regiduría de Ecología, Urbanismo y Movilidad, la medida de gobierno, "Urbanismo con perspectiva de género. El urbanismo de la vida cotidiana" para implementar políticas de planeamiento urbano, procesos y proyectos aplicando la perspectiva interseccional de género, poniendo la vida cotidiana y los cuidados en el centro de las decisiones urbanas. La medida define objetivos, metodologías a utilizar y una serie de actuaciones que generen el cambio del modelo urbano.

Siguiendo la actuación 3.1.2, que propone la elaboración de un mapa de ciudad que refleje la diagnosis perceptiva para poder responder adecuadamente a las necesidades de la vida cotidiana de los diferentes barrios, se realizaron, coordinados por los grupos de urbanistas feministas Col•lectiu Punt 6 y Equal Saree, marchas exploratorias y recorridos en algunos barrios y a partir de los cuales se realizó la redacción de un manual guía para realizarlas en otras zonas de

\footnotetext{
6 https://ajuntament.barcelona.cat/dones/sites/default/ files/documentacio/mesuradegovernurbanismeigenere 220317.pdf
} 
la ciudad. Paralelamente se ha hecho formación al personal técnico y político del consistorio.

Y siguiendo la actuación 3.1.4, que es la revisión con perspectiva de género del conjunto de elementos urbanos que componen el conjunto de mobiliario que se instala en el espacio público, así como la disposición en los diferentes espacios donde se sitúen, teniendo en cuenta las necesidades de las personas según el ciclo vital en que se encuentren, se realizó un proyecto para la colocación de asientos, bancos y sillas, en las aceras del distrito del Ensanche, en función de las redes cotidianas de las personas mayores. Este proyecto fue resultado de los presupuestos participativos del distrito.

A finales del año 2017 el ayuntamiento comenzó un proceso de participación para repensar colectivamente el Plan 22@ ${ }^{7}$ delante de los nuevos retos sociales, económicos y urbanos después de 17 años de su aprobación inicial. En este proceso participaron todos los agentes del barrio, ciudadanía, empresas y universidades, siendo uno de los aspectos principales el aumento de porcentaje de vivienda asequible que ha pasado del $10 \%$ al $30 \%$. El proceso se organizó en cuatro fases programadas, de octubre 2017 a mayo 2018: Información, diagnosis ciudadana, debate y retorno, incorporándose en la segunda los recorridos de reconocimiento cotidiano. Si bien en la metodología de los recorridos queda explícita la perspectiva de género, todo el proceso la ha incorporado, teniendo en cuenta una participación equilibrada de hombres y mujeres, así como la integración de los aspectos personales, productivos y reproductivos.

Los resultados cualitativos de los cuatro recorridos se realizaron a partir de los análisis territoriales de aspectos físicos, sociales y funcionales que influyen en la vida cotidiana desde las experiencias de las personas vecinas con una perspectiva de género interseccional, es decir, que tiene en cuenta la diversidad de sexo, género, edad, origen, diversidad funcional, entre otras. Siendo algunos de los resultados la necesidad de más vivienda pública, la percepción inseguridad en el 22@ por la falta de actividad a ciertas horas, la poca densidad y variedad de actividades; espacios públicos residuales ligados a los nuevos edificios corporativos que no están adecuados a las necesidades de vecinos y vecinas; la falta de equipamientos

${ }^{7}$ https://www.decidim.barcelona/processes/Repensem22a cotidianos y el descontento con las arquitecturas poco respetuosas con el patrimonio y el entorno, entre otras ${ }^{8}$.

Una de las estrategias fundamentales de la ciudad es el conjunto de proyectos que buscan hacerla más humana y más sana. Por un lado, es necesaria la disminución de los vehículos en la ciudad, ya que la cantidad de vehículos por $\mathrm{km}^{2}$ en Barcelona es superior a la de la mayoría de las grandes ciudades europeas y españolas, de lo que resulta una desproporción en el porcentaje de espacio público que se dedica al vehículo privado a motor en detrimento de la mayoría, así como unos niveles de contaminación, que superan lo permitido por la Unión Europea. Entre estos proyectos resaltan las experiencias denominadas "superilles", ya realizadas en Poble Nou y en Sant Antoni, que consisten en la limitación del tráfico pasante al tiempo que se adecúa el espacio público vehicular para usos ciudadanos. La singularidad de esta propuesta de peatonalización es que se trabaja sobre la estructura urbana del siglo XIX que ya fue pensada para la movilidad en máquinas frente a las actuaciones llevadas a cabo previamente en tejidos urbanos preindustriales y que los espacios de paso se convierten en espacio de juego y estancia. Esta experiencia se complementa con la recuperación de chaflanes de los que se retiran los vehículos y pasan a ser espacios de encuentro vinculados a centros educativos. Estas intervenciones se enmarcan, también, en el Pla de Joc a l'espai públic amb l'objectiu 2030, que se ha realizado sobre la pregunta de qué ofrece la ciudad y el entorno urbano para las personas de diferentes edades, y especialmente para la infancia. Se ha tomado los espacios de juego infantil como elemento estructurador urbano actual y futuro. Una ciudad amiga de la infancia pone desde el urbanismo el foco y la acción en la creación de entornos jugables, es decir, seguros e inclusivos.

La renaturalización de la ciudad es una estrategia que se enlaza con las anteriores y que se basa en un pensamiento ecofeminista, según el cual las personas somos ecodependientes e interdependientes, por lo que los proyectos han de tener en cuenta las necesidades ecosistémicas. Siguiendo estas ideas se ha proyectado la plaza de las Glorias, el nuevo gran centro urbano verde que a mediados de 2019 se haya en un $50 \%$ de su ejecución. Dentro de

\footnotetext{
${ }^{8}$ https://www.decidim.barcelona/processes/Repensem22a
} 
estas propuestas se encuentra el proyecto de humanización y naturalización de la avenida Meridiana para que deje de ser una gran cicatriz provocada por una autopista urbana y pase a ser un eje verde. En el marco de este proyecto se han identificado ejes transversales de gran potencial de conectividad entre los barrios a ambos lados de la vía y que se han trabajado participativamente para que el diseño responda a las necesidades cotidianas de las personas que habitan el territorio.

Los proyectos para la rehabilitación de espacios públicos comenzados en el mandato 20152019 han contado con procesos participativos en los que se ha incorporado la perspectiva de género, como la plaza dels Porxos en el Distrito de Sant Martí coordinado por Equal Saree en 2019 o la Plaza Soller, en el Distrito de Nou Barris, coordinado por el Col-lectiu Punt $6^{9}$ en 2016 y que ha sido la base del proyecto ganador del concurso realizado por Berta Barrio y Josep Peraire que se ha comenzado a construir en 2019.

\section{Santa Coloma de Gramenet, equipamiento y espacios públicos desde la perspectiva de género y los feminismos}

La política urbana de un gobierno feminista, como el de Santa Coloma de Gramenet encabezado por la alcaldesa Nùria Parlon, y con una composición política paritaria $50-50$, se distingue por pensarse desde el reconocimiento de la diferencia y priorizando la igualdad. La alcaldesa ha estado acompañada en el mandato 2015-2019 por mujeres referentes en políticas de género y feminismos como Gemma Bellvehí, Inma Moraleda, Maribel Cárdenas y yo misma.

Santa Coloma de Gramenet es la novena ciudad de Cataluña por población, con 118.000 habitantes provenientes de 117 países; la renta media familiar disponible es de $24.935 €$ laño, situándose entre las diez más bajas de la región. La superficie total de $7,5 \mathrm{Km}^{2}$, de los cuales $5 \mathrm{Km} 2$ son urbanos y ya están urbanizados, y en una gran proporción el territorio es orográficamente accidentado. La ciudad creció exponencialmente entre 1945 y 1975 , debido a las migraciones internas del estado español derivadas de la guerra civil, pasando en 30 años de menos de 20.000 a 140.000 habitantes, y como resultado de la velocidad del crecimiento la ciudad afronta continuos procesos de mejora y rehabilitación en todas las escalas, desde las alcantarillas a los espacios públicos y edificios. En ese proceso vertiginoso la ciudadanía fue agente activa en la construcción de la ciudad, lo que creó ciertas deficiencias en cuanto a la calidad de la edificación, al planeamiento (estructura urbana de grandes manzanas, con calles con mucha pendiente, parcelario de grano muy pequeño...) y a la falta de equipamientos, como suele pasar en las ciudades o barrios en que la gente auto produce su hábitat en que lo más difícil es la parte pública de la misma; sin embargo, por otro lado, las dificultades ligadas a la ciudad hecha a mano generó una sociedad muy combativa y organizada que fue fundamental a partir de la recuperación de la democracia en las ciudades con los primeros comicios en 1979. La situación geográfica de la ciudad de centralidad en el Área Metropolitana de Barcelona, a 20 minutos en metro de Plaza Cataluña (centro de la ciudad de Barcelona) la hace un lugar de centralidad y oportunidad.

Estas características físicas y sociales hacen de la ciudad un laboratorio perfecto del futuro ya que plantea cuestiones cruciales en un planeta antropizado y superpoblado, en el que la mayoría de la población vive en ciudades no planificadas y con escasos recursos, y en el que las mujeres sufren las peores consecuencias por falta de seguridad, falta oportunidades de acceso a recursos, y menores ingresos. Las preguntas planteadas en esta situación ¿Cómo mejorar y transformar una ciudad ya construida?, ¿Cómo hacer políticas urbanas que sean inclusivas?, ¿Cómo trabajar para que la ciudad sea un soporte adecuado para vidas cotidianas con tantas dificultades?

La ciudad es pensada desde la proximidad, desde la experiencia cotidiana, con una lógica inductiva, es decir, del detalle al todo, tal como recomendaba Jane Jacobs en su libro Muerte y vida de las grandes ciudades de 1961. Los proyectos de ciudad están pensados desde la cercanía, la atención y cuidado por cada detalle y por cada persona marcan cada acción política. En términos de urbanismo, no solo se actúa en los proyectos de impacto, sino que las pequeñas actuaciones como el mantenimiento y la limpieza

\footnotetext{
${ }^{9}$ Más información en http://www.punt6.org/proyectos-de-punt-6/
} 
se sitúan en igualdad de condiciones. No se desechan los grandes proyectos o intervenciones, sino que se equiparan en valoración.

Un proyecto emblemático y pionero en políticas feministas es la rehabilitación del antiguo edificio que ocupaba la fábrica Ciba, posteriormente juzgado e instituto, para ser el nuevo Centro de recursos para mujeres, que amplía su alcance y su ambición, siendo un centro de referencia feminista y de género. Se trata de una propuesta de un nuevo tipo de equipamiento en el que la producción y la reproducción, lo personal y lo socio-comunitario-político puedan conjugarse en el tiempo y en el espacio, basada en la economía social, colaborativa y feminista.

El proyecto se ha abordado por fases de una manera contenida, es decir, que con actuaciones mínimas se recupere progresivamente el edificio y se genere un condensador social. Será el centro de las políticas públicas con perspectiva de género siguiendo la idea de la zona de recursos para mujeres. La idea principal de un centro o zona de recursos para las mujeres es integrar las cuatro esferas de la vida de manera que las mujeres no queden excluidas, especialmente, de la producción por las obligaciones derivadas de las tareas de género asignadas. Por ello, se proponen espacios de formación y espacios para la aplicación de los conocimientos adquiridos a través de la generación de propuestas cooperativas y colaborativas de empleo, al tiempo que se ofrecerán espacios para las personas dependientes, especialmente niñas, niños y jóvenes.

El proyecto de remodelación de la Plaza BaróJardines José Beruezo ha sido co-creado con las niñas y niños de Santa Coloma de Gramenet $y$ el enfoque feminista ha sido clave, tanto en el desarrollo del proceso participativo como en la redacción del proyecto técnico de la plaza, integrando los valores de la corresponsabilidad, el respeto a las diversidades y la igualdad de género. La plaza no era muy utilizada en un barrio con gran densidad, que llevaba a un uso excesivo de la plaza del Olimpo que se encuentra próxima. Por esto surgió la necesidad de analizarla y repensarla conjuntamente con sus usuarias y usuarios principales que son niños y niñas del entorno, buscando generar una red de plazas en el barrio de soporte a la vida cotidiana. El proceso de participación y co-diseño contó con tres etapas: la inicial en la plaza, la mañana de dos sábados en noviembre de 2016 , invitando a usuarias y usuarios a realizar un diagnóstico y propuesta de usos; la segunda, con niños y niñas de quinto curso de la Escuela Torrebaldovina, en el que se tomó como base el trabajo anterior, que hicieron propuestas de diseño en equipos de dos niñas y dos niños; la tercera y última fase de proyecto fue la realizada por las arquitectas de Equal Saree ${ }^{10}$ en la que conjugaron en una única propuesta los resultados y esta fue explicada a los niños y niñas de la escuela.

Los objetivos perseguidos han sido: repensar un espacio lúdico de juego libre, inclusivo y diverso donde se visibilicen y normalicen las tareas de cuidado en el espacio público; tener en cuenta las emociones y la diversidad de experiencias de "todas las personas" reconociendo el bienestar como un derecho de ciudadanía; y potenciar el trabajo colectivo y la reflexión sobre el espacio como herramientas educativas, valorando el bien común por encima de los intereses individuales. El resultado es una plaza colorida y diversa que visibiliza y facilita los cuidados.

\section{Otras experiencias: Girona, Garraf y Olessa}

El proyecto de las ágoras cotidianas en Girona, liderado por la entonces regidora Isabel Salamaña, comenzó en 2002 como resultado de demandas vecinales para la mejora de zonas de juegos infantiles que había comenzado en 1994 con el análisis y evaluación de las zonas de juegos infantiles, y en 1995 se comenzó la renovación progresiva de las 52 áreas de juegos de la ciudad. Las zonas de juego son una forma eficaz de recuperar y potenciar los espacios de encuentro y diversidad en la ciudad. Se partió del principio que las calles, aceras, esquinas, plazas, parques como espacios públicos que usamos para desplazarnos congregan diversidad y pueden hacer interactuar a las personas entre sí. Por ello, el proyecto de ágoras cotidianas buscó mejorar su interdependencia, reforzando itinerarios para potenciar encuentros y relaciones.

${ }^{10}$ Más información sobre el proyecto http://equalsaree.org/ blog/2017/12/02/fem-dissabte-a-placa-baro/ acceso 15-6-2019 
Los objetivos del proyecto de "ágoras cotidianas" fueron la colocación de zonas de juego infantil cerca de las guarderías, jardines de infancia y escuelas primarias, a una distancia de $200 \mathrm{~m}$ creando itinerarios de vida cotidiana que aproxime espacios, potenciando en la medida de lo posible la mezcla de usos; promoviendo las zonas de juego en los espacios públicos para posibilitar el encuentro, incrementar las redes sociales y facilitar las relaciones de vida cotidiana; y haciendo suyo el principio de la ciudad plural, que con cuantos más recorridos cuente más rica y plural será. La implantación de las zonas de juego permite crear pequeñas centralidades urbanas que refuercen el espacio público como lugar de proximidad de vida cotidiana, siendo las personas más beneficiadas niñas, niños y la gente mayor (SALAMAÑA, 2012).

La escala regional la encontramos en el estudio de las urbanizaciones de baja densidad de la comarca del Garraf, fue encargada al Col•lectiu punt 6 por el Consell Comarcal para que se siguiera la investigación académica financiada por la beca Recercaixa, Rehabilitación de urbanizaciones residenciales monofuncionales de baja densidad (Muxi, 2013), en la que se aportaban alternativas para mejorar la vida cotidiana en las urbanizaciones residenciales desde una perspectiva de género. El estudio realizado en 2017 fue enfocado en la necesidad de vincular tres dimensiones aplicando la perspectiva de género, es decir, vincular escalas y dimensiones, lo físico, lo social y lo legal-urbanístico. La primera fue la unidad residencial en la que las principales problemáticas detectadas son la falta de adecuación de las tipologías a los grupos de convivencia, la calidad constructiva y el estado de conservación, los problemas de suministros básicos, los altos costes de la tipología, diferentes situaciones de ilegalidad y viviendas vacías. La segunda, las personas que habitan en estas urbanizaciones, muchas veces no están registradas y en consecuencia se desconocen las situaciones socio-económicas en la que se hallan, sufren un alto grado de aislamiento y falta relación entre ellas. Además de falta de títulos legales de la propiedad, hay muchas personas con falta de recursos atrapadas en una casa con jardín y esta situación de aislamiento provoca una gran problemática de convivencia y de participación comunitaria con el municipio al que pertenecen. Por último, las problemáticas detectadas y vinculadas al entorno están relacionadas a problemas con la situación urbanística y sus condiciones, la monofuncionalidad y la falta de equipamientos, el gran déficit en la calidad del espacio público, la falta de redes cotidianas que vinculen las actividades, las opciones de transporte público viable y con graves problemas de contaminación medio ambiental y paisajística del territorio natural y artificial. Las dificultades que afrontan las personas en su día a día y la gran complejidad que presenta el entorno por sus diferentes casuísticas y condiciones territoriales hacen necesario abordar las actuaciones desde una mirada integradora. No se trata sólo de un modelo insostenible en términos territoriales y energéticos, sino también insostenible para la vida de las personas, en relación a sus tiempos y al derecho a desarrollar sus propias vidas.

Las auditorías de género son una herramienta que permiten elaborar recomendaciones en la redacción de los POUM para cumplir con el requerimiento del Informe de impacto de género que exigen las leyes catalanas de urbanismo, el Text refós de 2012 i la LLei d'igualdat efectiva entre dones i homes de 2015, así como la Ley orgánica de igualdad entre hombres y mujeres del 2007. Es en este contexto que el equipo redactor del POUM de Olesa de Montserrat, Amador Ferrer e Isidre Roca han incorporado a las técnicas de Punt 6, Adriana Ciocoletto y Blanca Valdivia, en la redacción del plan para incorporar criterios de género en todo el proceso y no solamente como informe final.

La auditoría ha propuesto una evaluación transversal incorporando diferentes agentes y conocimientos. Para ello uno de los primeros requerimientos es que el equipo técnico del ayuntamiento vinculado a la redacción del plan reciba formación específica desde la perspectiva de género.

Al encargo inicial del ayuntamiento se ha sumado el requerimiento de la Diputació de Barcelona para que se incorporen criterios de salud al urbanismo, para lo que contaron con expertas en salud y feminismo del Centre d'Anàlisi i Programes Sanitaris (CAPS), resultando una Auditoria Urbana de Género y Salud. La base de esta incorporación son los criterios definidos en la "Evaluación del impacto en salud" (AIS en su sigla en catalán) que tienen como objetivo maximizar los efectos positivos y minimizar los negativos de nuevas iniciativas, como es en este caso el POUM.

Como ya se ha dicho, esta auditoria ha acompañado toda la redacción del plan, desde el pre avance de plan hasta la aprobación inicial, haciendo posteriormente un seguimiento a partir del informe de impacto de género, realizando un trabajo transversal entre diferentes áreas del ayuntamiento, el equipo redactor del plan y un 
grupo de mujeres vecinas de perfiles diversos con quienes se ha trabajado sus experiencias de vida cotidiana desde una perspectiva de género y salud.

Se ha trabajado desde las experiencias cotidianas de las mujeres, es decir, poniendo en el centro del análisis y las propuestas la vida y los cuidados, que dan un conocimiento más complejo de la realidad debido a la doble o triple jornada que continúan desarrollando mayoritariamente las mujeres en nuestra sociedad, siempre teniendo en cuenta las diversas necesidades de las personas de acuerdo con las diferencias de género, edad, origen y cultura, situación socio-económica y diversidad funcional, entre otras.

El equipo técnico encargado de la auditoría ha analizado la documentación e información disponible, ha realizado visitas y recorridos por el territorio, dinamizado talleres con las mujeres y realizado reuniones tanto con el equipo redactor como con el equipo técnico del propio ayuntamiento para poder elaborar el diagnóstico y las recomendaciones hasta llegar a la evaluación e informe final.

\section{Conclusiones}

Esta revisión, mayoritariamente focalizada en Barcelona, demuestra todo lo que se ha avanzado en la incorporación de la perspectiva de género y el urbanismo feminista en diversas ciudades catalanas, aunque no podamos decir que es una aproximación mayoritaria, sí que ya no causa extrañeza su implementación y cada vez es más comprendida. Aunque aún hace falta una mayor incidencia desde la formación universitaria en la que aún no se ha incorporado de manera integrada y transversal.

Las estrategias para la implementación de políticas de género y feministas del gobierno de la ciudad de Barcelona han sido hechas de manera integrada y multiescalar. Desde las normativas a la incorporación de la participación ciudadana con perspectiva de género, al tiempo que desde otros ámbitos como la cultura o los eventos de representación política se ha buscado la paridad de representación, dando un mayor protagonismo a las mujeres para compensar los años de invisibilidad de las aportaciones de las mujeres. Sirva como demostración la elección de mujeres como pregoneras de la Fiesta de la Mercé de 2017, 2018 y 2019, Marina Garcés, Leticia Dolera y Manuela Carmena respectivamente.
Lo especialmente destacable en las políticas reseñadas, es que se trata de cambios profundos y como tales atraviesan todas las esferas de la acción política, todos los servicios, buscando la interrelación entre los mismos. La realidad comprendida y trabajada a partir de cajones estancos va dejando paso a un entrelazamiento y a un cambio de prioridades, poniendo la vida cotidiana y los cuidados en primer plano. Estos cambios, que están enraizados en los movimientos y luchas feministas de décadas, no son acciones solitarias de gobierno, sino reflejo de una sociedad que aspira a la igualdad real.

\section{Bibliografía}

BASTARDES, Montserrat (2018): Les escoles bressol de l'Ajuntament de Barcelona, Memòria històrica 1932-2007. Ajuntament de Barcelona, Barcelona, 2018.

BLANCHARD, Clara (2018): "L'escola bressol municipal número 100. Barcelona disposa d'una de les xarxes de llars d'infants públiques més grans d'Europa" en El País, edición Catalana, Barcelona, 26 de diciembre de 2018.

https://cat.elpais.com/cat/2018/12/26/catalunya/ 1545846163 744787.html

acceso 11-08-2019

BofILL, Anna. (2013): "Derecho de las mujeres a la ciudad" en Urbanisme Inclusiu, Barcelona.

https://urbanismeinclusiu.files.wordpress. com/2013/03/el-derecho-de-las-mujeres-a-la-ciudad anna-bofill.pdf acceso 12-6-2019

FALAGAN, David (2019): Innovación en vivienda asequible Barcelona 2015-2018. Ajuntament de Barcelona, Barcelona, 2019

LACOL \& LA CIUTAT INVISIBLE (2018): Habitar en comunidad. La vivienda cooperativa en sesión de uso. Fundación Arquia, Barcelona, 2018.

MAgro Huertas, Tania (2014): Hacia la ciudad inclusiva. Prácticas sociales urbanas en Barcelona 1969-1979. Tesis Doctoral (no publicada), Director. Josep Maria Montaner. ETSAB-UPC

https://www.tdx.cat/handle/10803/277359\#page=1 (último acceso 10-08-19)

Muxi Martínez, Zaida (2013): Postsuburbia. Rehabilitación de áreas monofuncionales residenciales de baja densidad. Barcelona, España: Ed. Comanegra.

- \& Magro Huertas, Tania (2009): "Women as city builders coming out of Urban Social Movements" en The New Urban Question - Urbanism beyond Neo-Liberalism. The $4^{\text {th }}$ International Conference of the International Forum on Urbanism (IFoU). Amsterdam / Delft.

Ortiz Escalante, Sara (2019): Planning the everyday/ everynight. A feminist participatory action research with women nightshift workers. Doctoral Thesis. University of British Columbia. 
https://open.library.ubc.ca/clRcle/collections/ ubctheses/24/items/1.0379046

SALAMAÑA I SERRA, Isabel (2012): «Les àgores quotidianes».

En Blanca G. Valdivia y Adriana Ciocoletto (coordinadoras) Estudios urbanos, género y feminismo:
Teorías y experiencias. (pp. 25-37). Barcelona, España, Col•lectiu Punt 6.

Torns, Teresa \& BorRAs, Vicent \& MoREno, Sara \& RECIO, Carolina (2006): Les politiques del temps: un debat obert. Regiduria Nous Usos Socials del Temps. 